\title{
Finite Element Modelling of Shock Wave Propagation Over Obstacles
}

\author{
Josef Soukup ${ }^{1}$, František Klimenda ${ }^{1}$, Jan Skočilas ${ }^{2}$, Milan Žmindák ${ }^{3}$ \\ ${ }^{1}$ Faculty of Mechanical Engineering, J. E. Purkyne University in Usti nad Labem. Pasteurova 3334/7, 400 01 Usti nad \\ Labem. Czech Republic.josef.soukup@ujep.cz, frantisek.klimenda@ujep.cz \\ ${ }^{2}$ Czech Technical University in Prague, Faculty of Mechanical Engineering, Department of Process Engineering, Jan.Sko- \\ cilas@fs.cvut.cz \\ ${ }^{3}$ Faculty of Mechanical Engineering, University of Žilina, Univerzitná 1, Žilina 010 26, Slovak Republic, milan.zmin- \\ dak@fstroj.uniza.sk
}

\begin{abstract}
The current software allows to simulate the behavior of the technical objects on the pressure wave of detonation fumes, the spread of the air and the action on the construction, and on the people. It is possible to simulate the changed conditions and their effect on objects without that it would be necessary to make costly and time consuming tests. Modeling of the pressure wave belongs to the fluid flow interference and obstacles in the flow. This paper aims to develop simulation models based on the finite element method for the elasto-plastic wave propagation from the explosion of a spherical explosive. First, it is analyzing the propagation of pressure waves at a contact explosion with a perfectly rigid surface, which represents the face of the earth. Then it analyzed explosion contact with deformable surface. Finally, it is investigated propagation of pressure waves through the ditches around the perpendicular wall.
\end{abstract}

Keywords: Pressure wave, Shock wave, TNT

\section{Introduction}

Shock loading can be found in the interaction between flying object with path obstacle, effects of explosive detonation, irradiation of the material by laser working at pulsating regime and other processes during those the rapid or often abrupt energy release happened. Specified loading is characterized by short time duration, high shear rates compared to static and steady dynamic loading [1, 2]. However, the value of shear rate is not crucial characteristics of this type of loading. The most important characteristic is stress wave or stress pulse in the loaded body when the propagation of the stress should be described [3]. The stress waves transfer all the force impacts into the bodies and are distributed by finite velocity, which depends mainly on material properties and contact parameters $[4,5]$.

The shock wave is generated when the movement of the body or disturbance in the environment has high velocity (e.g. in the gas the velocity is higher than average velocity of molecules). The generation of the impulse wave can be approximately described by body or disturbance passing through material where the sound wave is originated. These sound waves are interpolated to shock wave, when the velocity of the sound source is supersonic velocity. By other words, the shock wave is formed, when the velocity of the gas flow is changing more than velocity of sound. In the location of that change, the sound wave are not allowed to proceed in the counter current direction. The pressure rises and the sound wave is generated. The material changes its properties by jump function (density, pressure, temperature, velocity, Mach number) at stepping front. The experimentally verified thickness of the stepping front of the shock wave (region of the jump change) is approximately only one order higher than mean free trajectory in the material [6]. In the ordinary atmospheric conditions the thickness of the stepping front of the shock wave is approximately one micrometer. The shock wave are not common sound waves. They are not periodic and the sharp rising of the pressure is significant for them [7, 8]. In the longer distance, the impulse wave can dissipate energy into the heating the air, therefore they are gradually transformed to the sound waves. After supersonic aircraft crossing, the characteristics sonic boom can be heard.

The aim of this paper is development of the simulation models based on the finite element method (FEM) for propagation of the elastic-plastic waves $[9,10]$ of sphere explosive detonation. Firstly, the propagation of the pressure wave at contact explosion with ideally rigid surface (which represents surface of the earth) is analyzed. Then the contact explosion with deformable surface is analyzed. Finally, the propagation of the pressure wave over the ditch and perpendicular wall is investigated.

\section{Governing equations}

The governing equations of the convection in conservative form for numerical solution arise from differential equations - equation of continuity, motion equation, energy equation, which can be written in the form (Navier-Stokes equation)

$$
\frac{\partial U}{\partial t}+\frac{\partial\left(E-E^{\prime}\right)}{\partial x}+\frac{\partial\left(F-F^{\prime}\right)}{\partial y}+\frac{\partial\left(G-G^{\prime}\right)}{\partial z}=J
$$

The followed equation represents comparison of the continuity equation with governing form

$$
\frac{\partial \rho}{\partial t}+\frac{\partial \rho c_{x}}{\partial x}+\frac{\partial \rho c_{y}}{\partial y}+\frac{\partial \rho c_{z}}{\partial z}=0
$$

where $U=\rho, E=\rho c_{x}, F=\rho c_{y}, G=\rho c_{z}$, $E^{\prime}=F^{\prime}=G^{\prime}=0$ a $J=0$.

By the same way, the motion equations or energy 
equation can be re-written. For the special case of the inviscid fluid, Navier-Stokes equation are simplified and identified as Euler equations of fluid flow. These equations have the same form in principle, but the tensors of stresses and heat fluxes are zero.

$$
U=\left(\begin{array}{c}
\rho \\
\rho c_{x} \\
\rho c_{y} \\
\rho c_{z} \\
\rho e
\end{array}\right), E=\left(\begin{array}{c}
\rho c_{x} \\
\rho c_{x}^{2}+p \\
\rho c_{x} c_{y} \\
\rho c_{x} c_{z} \\
\rho h_{0} c_{x}
\end{array}\right), F=\left(\begin{array}{c}
\rho c_{y} \\
\rho c_{y} c_{x} \\
\rho c_{y}{ }^{2}+p \\
\rho c_{y} c_{z} \\
\rho h_{0} c_{y}
\end{array}\right), G=\left(\begin{array}{c}
\rho c_{z} \\
\rho c_{z} c_{x} \\
\rho c_{z} c_{y} \\
\rho c_{z}^{2}+p \\
\rho h_{0} c_{z}
\end{array}\right)
$$

where $c_{x}, c_{y}, c_{y}-$ velocity field components, $p-$ pressure, $\rho$ - fluid density, $e$ - energy, $h_{0}$ - enthalpy.

In the flow tensors, the total enthalpy instead of the energy is defined in the Euler equation according to followed equation

$$
\rho e+p=\rho\left(c_{v} T+\frac{c^{2}}{2}+\frac{p}{\rho}\right)=\rho\left(c_{p} T+\frac{c^{2}}{2}\right)=\rho h_{0}
$$

where $T$ - temperature, $c_{v}$ - specific thermal capacity at constant volume, $c_{p}$ - specific thermal capacity at constant pressure and $c$ - velocity.

There are six unknown quantities in the five equations. The system is completed by equation of state for the ideal gas.

$$
p=(\gamma-1) \rho e
$$

where $e$ - specific internal energy of the gas and $\gamma-$ is the adiabatic index.

There are several equations of state for gas, but most of the gases are described by equation of state for ideal gas. For liquid in the simplest form, the simple volume equation of state is applied

$$
p=K\left(\frac{\rho}{\rho_{0}}-1\right)
$$

where $K$ - material volume module, $\rho_{0}$ - base density.

\section{Numerical simulation}

\subsection{Rigid ground}

At the beginning, the propagation of the pressure wave in the space within the obstacles with ideal rigid and deformable ground is assumed. In the Figure 1, the scheme of the geometry is shown. The half part of the model is investigated. The Euler mesh represents the block having the dimensions $9000 \times 2000 \times 3000 \mathrm{~mm}$ and it is uniformly distributed by hexagonal elements. The dimensions of the each element are $50 \times 50 \times 50 \mathrm{~mm}$. Total number of the elements is 432000 . The half part symmetrical model with plane of symmetry $x z$ is investigated (Fig. 2). At outer boundaries of the investigated region, the boundary conditions are defined with respect to smooth distribution of the pressure drop to the external part
The differential form of the Euler equations is:

$$
\frac{\partial U}{\partial t}+\frac{\partial E}{\partial x}+\frac{\partial F}{\partial y}+\frac{\partial G}{\partial z}=0
$$

of the region and they eliminate the reflection of the wave at the model boundaries (Flow out).

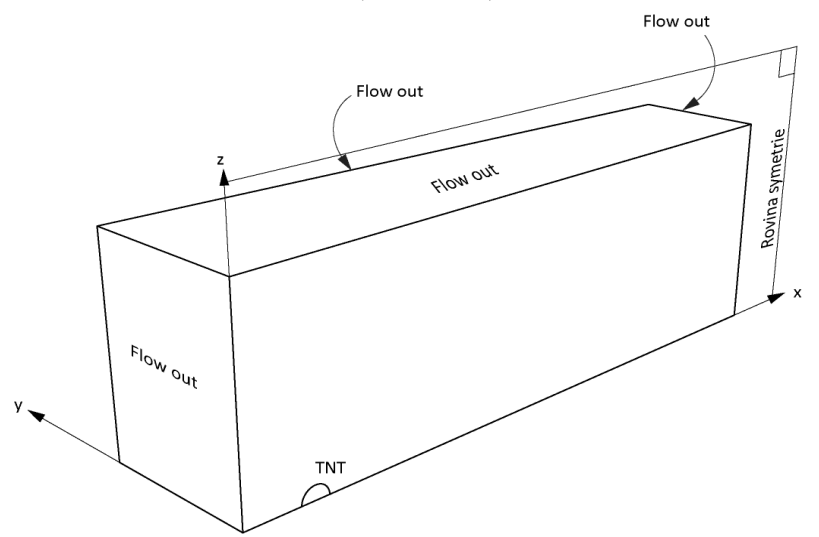

Fig. $13 D$ scheme of the investigated model

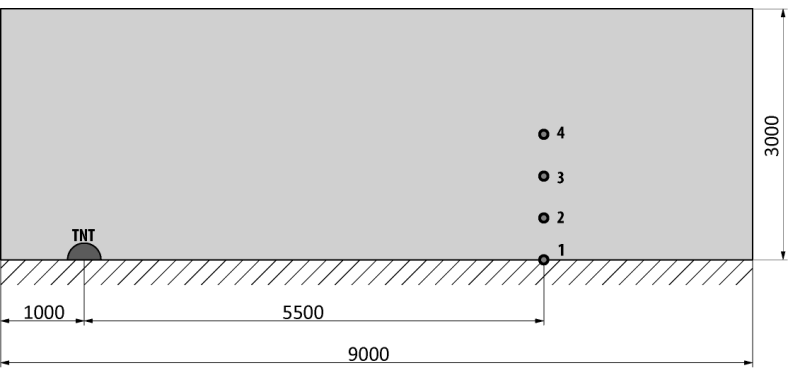

Fig. 2 Scheme of the model without obstacle with ideal rigid ground

The blue area represents Euler mesh. The position of the explosive is marked by red semicircle. The considered obtaining of the basic values of pressure during explosion of described explosive and for propagation pressure wave in the region with contact explosion with ideal rigid surface, which represents earth ground. The material models of the air and TriNitroToulene (TNT) is described in [8] and the material of the earth ground is based on a compaction Equation of State (EOS) which is used to describe the unloading wave as function of both density and pressure [11].

The check measuring points are considered at distance $5500 \mathrm{~mm}$ from the place of explosion initialization. The time pressure dependence was measured in these points. The displacement of the particular points is $500 \mathrm{~mm}$ in the $z$ axis direction. The pressure trends at each check measuring points are presented in the figure 3 . The pressure wave is spreading from explosion initialization point in form of semi-sphere. With regards to relatively small distance between measuring points, the pressure trends explosive had weight $7 \mathrm{~kg}$ of TNT. This model served for 
are nearly similar. The small differences between trends are caused by various displacement of measuring points from explosion initialization point. However the reflection of the pressure wave stepping front is not presented, the time trends of pressure in the particular places have expected shape. Each curve has only one maximum, which represents instant of the transition of the stepping front of the pressure wave across the check measuring point.

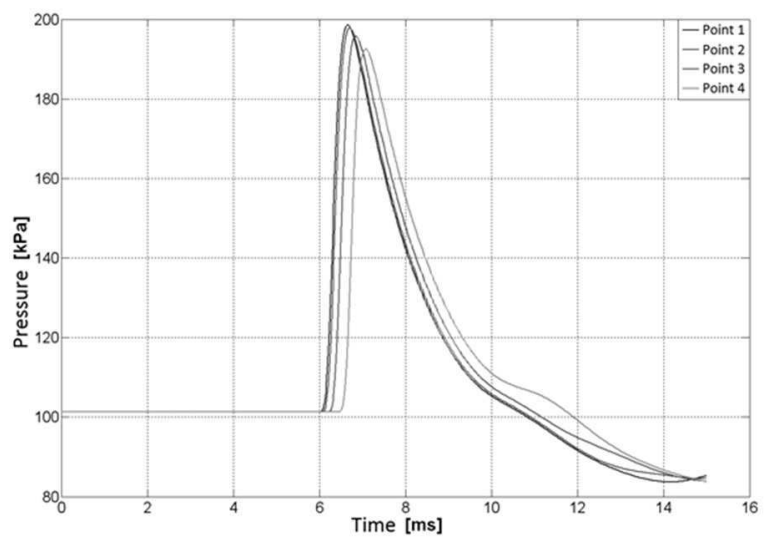

Fig. 3 The course of pressures in the investigated points, case without obstacle; rigid ground

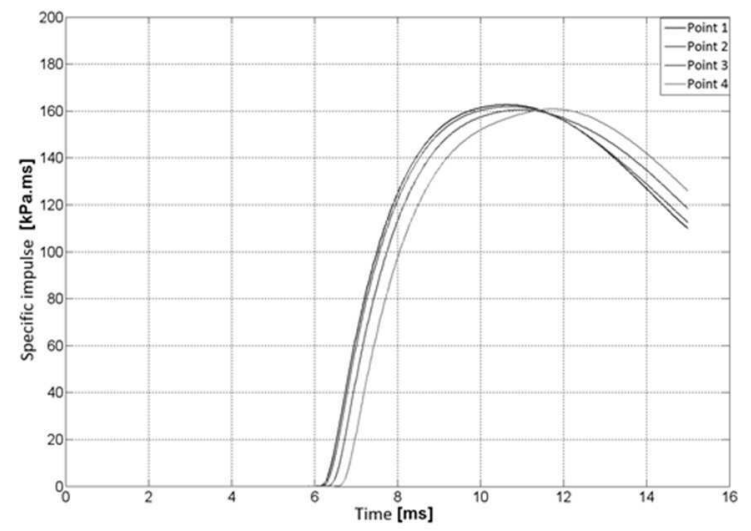

Fig. 4 Specific the course of impulse in the particular investigated points, case without obstacle

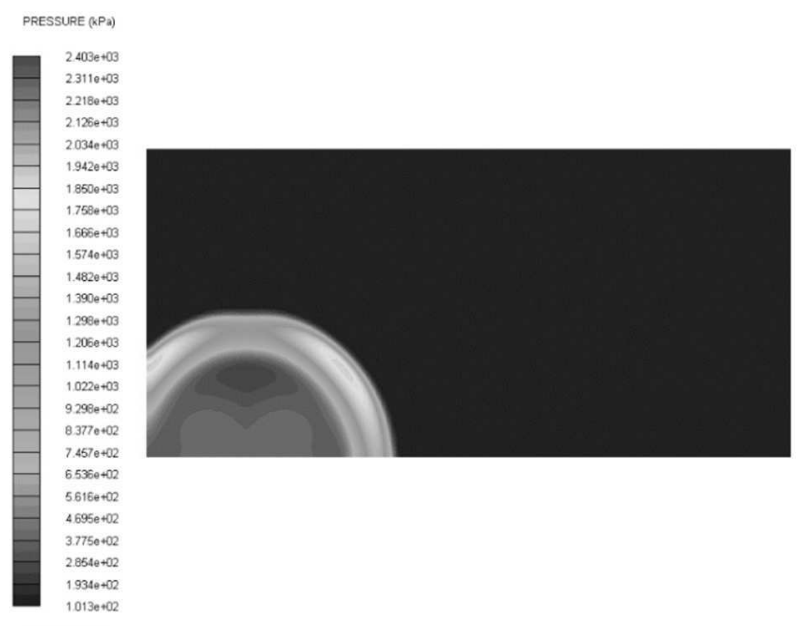

Fig. 5 Shock wave contours without obstacle for time $0.5, \mathrm{~ms}$

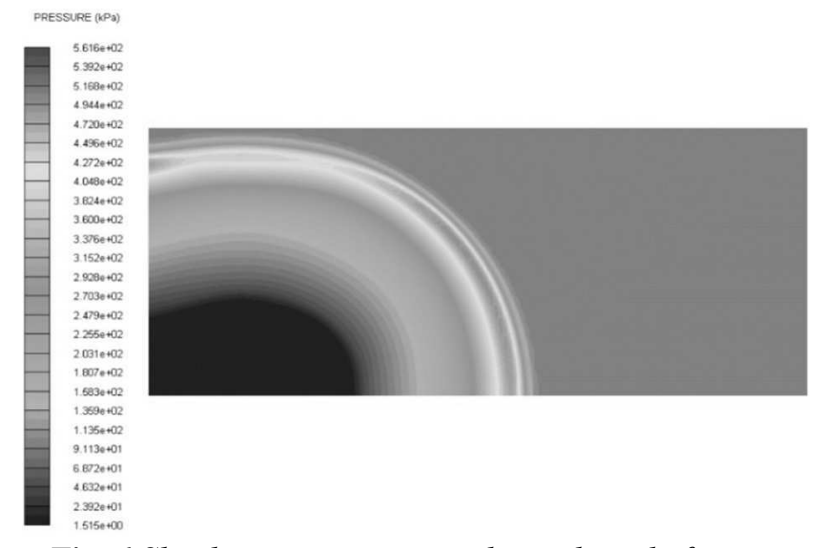

Fig. 6 Shock wave contours without obstacle for time $2.0 \mathrm{~ms}$

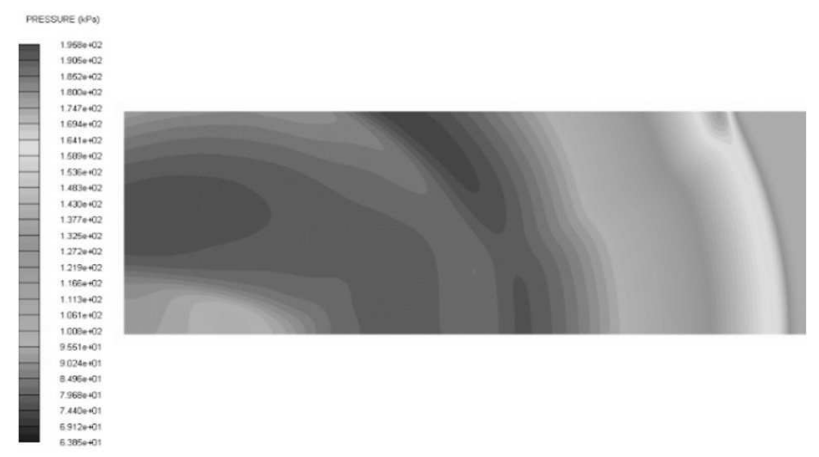

Fig. 7 Shock wave contours without obstacle for time $10.0 \mathrm{~ms}$

The figure 4 represents the specific impulse trends in the particular investigated points. Maxima are for all measured points approximately similar $160 \mathrm{kPa} . \mathrm{ms}$. The free distribution of the pressure wave in the space without obstacle is presented in the figure 5, 6 and 7. It is obvious, that there is an absence of the pressure wave reflection at border of the investigated region, where the boundary conditions „Flow out“" are defined.

\subsection{Deformable ground}

In the Figure 6, the scheme of the model for calculation of the pressure wave propagation in the space without obstacles is shown. The blue area represents Euler mesh filled by air and brown area represents Euler mesh filled by ground. The position of the explosive is marked by red semicircle. The considered explosive had weight $7 \mathrm{~kg}$ of TNT. This model served for comparison of the values of pressures during explosion of described explosive and for propagation pressure wave in the region with contact explosion with deformable surface, which represents earth ground.

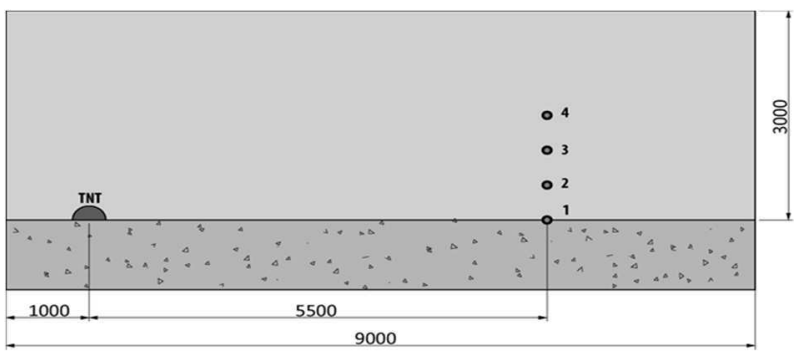

Fig. 8 Scheme of the model without obstacle with deformable ground 


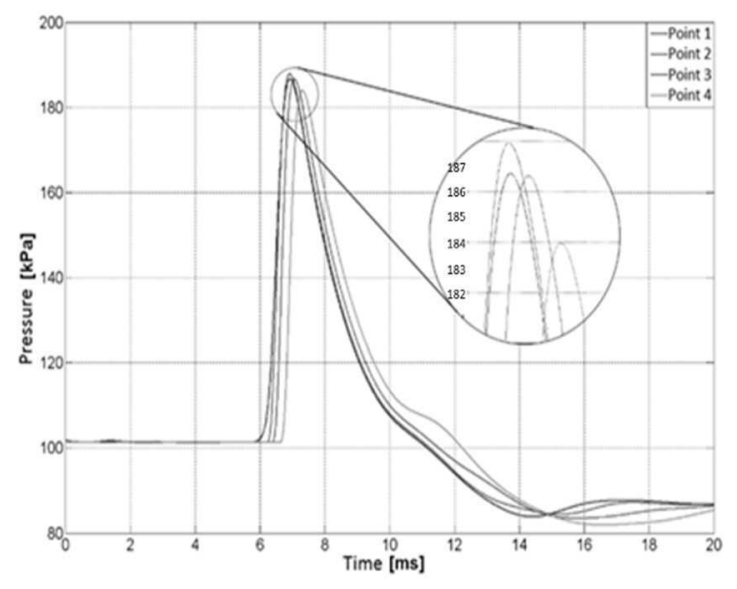

Fig. 9 The course of pressures in the investigated points for case without obstacle
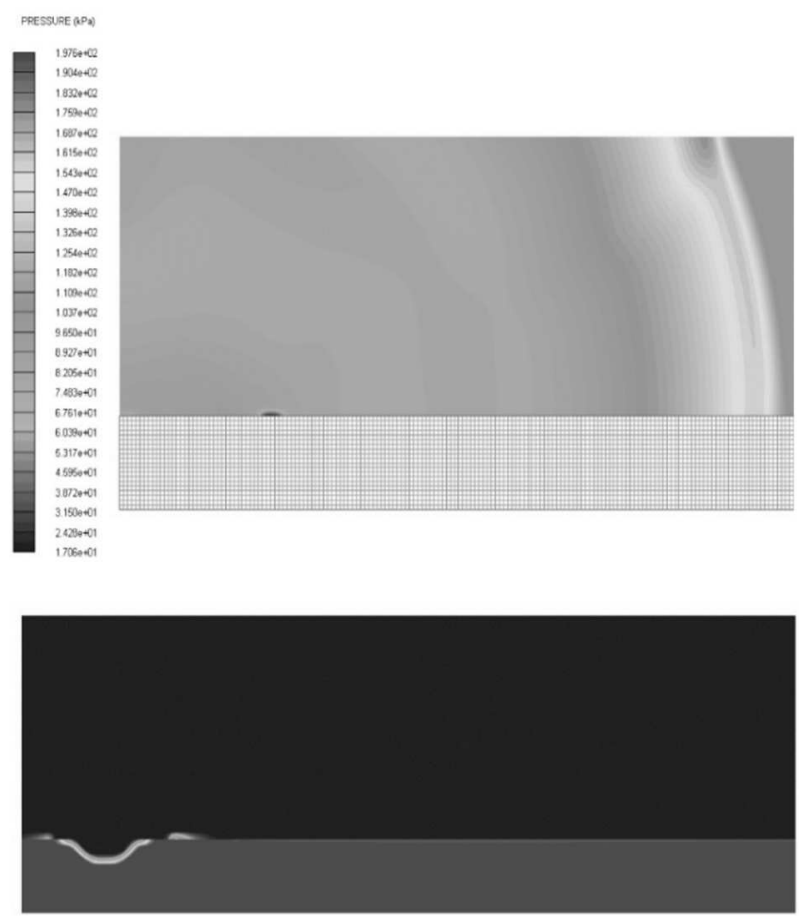

Fig. 10 Shock wave contours for free propagation and timeframe of crater generation in time $10 \mathrm{~ms}$

The half part of the model is investigated. The Euler mesh represents the block having the dimensions $9000 \times$ $2000 \times 4000 \mathrm{~mm}$ and it is uniformly distributed by hexagonal elements. The dimensions of the each element are $50 \times 50 \times 50 \mathrm{~mm}$. Total number of the elements is 576000 . The half part symmetrical model with plane of symmetry $x z$ is investigated (Fig. 8). At outer boundaries of the investigated region, the boundary conditions are defined with respect to smooth distribution of the pressure drop to the external part of the region and they eliminate the reflection of the wave at the model boundaries (Flow out). The locations of the check measuring points are same as in the case with ideally rigid ground. The pressure trends at each check measuring points are presented in the figure 9. The maxima of the particular trends are zoomed in detail. It is obvious, that maximal pressure is not present in the point 1 , compare to the case with rigid ground. This difference is probably caused by dumping effect of the ground acting on the impulse wave.

In the figure 10, the impulse wave contours is shown, which is generated by pressure wave propagated by free space without obstacle, considering deformable ground and timeframe of the crater generation, which is produced by effect of the surface erosion.

\subsection{Results comparison}

Both previous cases represent free propagation of the pressure wave, which was generated by contact explosion. The difference between them is boundary condition types, which defines earth ground. In the first case, the ground is considered as ideally rigid (un-deformable) surface. In the second case, the earth ground is defined by Euler domain with material properties, which represents soil, it is in fact the deformable surface. For both cases the check measuring points are placed at same locations for mutual comparison of the cases. In the figure 3 and 9, the pressure trends in the particular check measuring points are presented. It is obvious, that in all points the maximum values of the pressures are smaller for case with deformable ground.

In the first case, there is the ideal reflection of the pressure wave and all energy, which is carried by pressure wave hits the measuring points with full impact. In the second case, the pressure wave which has been released by explosion, reach the interface ground-air, and the partial reflection of the pressure wave from this interface back to the air happens. Second part is distributed into the soil in the form of tensile stress wave. The tensile stress acts within the soil, causes the expansion and fragmentation of the soil. The particular fragments are consequently ejected by effect of high pressure produced by explosion. The crater is originated by this effect in the initial location of the explosive. This division of the explosion energy results in shock wave, which is distributed in air, and has lower intensity. This is demonstrated by lower maximum value of the pressure in particular points. It can be found from the figures, that in the second case, the impulse wave arrived later to the investigated point. However this difference is minimal.

\subsection{Propagation of the pressure wave over ditch}

In the Figure 11, the scheme of the model for calculation of the pressure wave propagation in the space with presence of ditch is shown. The blue area represents Euler mesh. The position of the explosive is marked by red semicircle. The considered explosive had weight $7 \mathrm{~kg}$ of TNT. This model served for obtaining of the essential values of pressure during explosion of described explosive and for propagation pressure wave also in the region of the ditch. Model represents contact explosion with ideal rigid surface, which represents earth ground. The walls of the ditch are also rigid. The spatial arrangement and boundary condition definitions are similar as previous cases and it is shown in the figure 11.

The distance of the ditch from location of the explosive is $5000 \mathrm{~mm}$. The ditch depth is $1500 \mathrm{~mm}$ and width $1000 \mathrm{~mm}$. At distance of $5500 \mathrm{~mm}$ from location of the explosive (in the middle of the ditch) the check measuring 
points are considered, in which the time pressure trend was recorded. The displacement of the particular points is $500 \mathrm{~mm}$ in the $\mathrm{z}$ axis direction. By the same method as used in the case of free propagation, this model is uniformly divided by hexagonal elements with dimensions $50 \times 50 \times 50 \mathrm{~mm}$. Total number of the elements is 456000 .

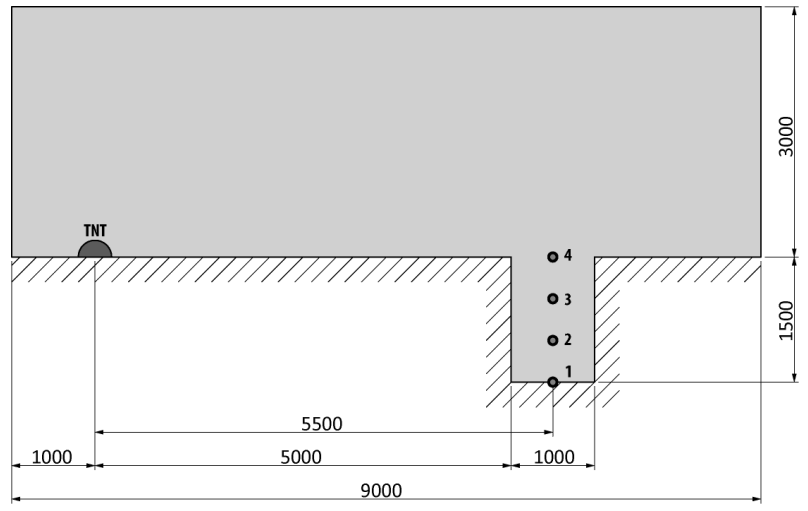

Fig. 11. Scheme of the model

In the figures $12,14,15$ and 16 , the pressure trends in particular check measuring points in the ditch and the propagation of the pressure wave contours in the investigated region are shown. The stepping front of the wave reached the ditch boundary approximately at $6 \mathrm{~ms}$ after initialization. From this moment, the stepping front of the pressure wave bends and leaks into the ditch. The first check measuring point affected by pressure wave is number 4 , points 3, 2 and finally 1 follow. After leakage of the pressure wave into the ditch, there are multiple reflection from ditch wall and bottom. The pressure trends exhibit several local maxima and the time incidence of increased pressure is longer, compared to the case with free space propagation without obstacle. This effect is the most demonstrated in the bottom layers of the ditch, where the higher superposition of the pressures appeared.

The figure 13 represents the specific impulse course in the particular investigated points. From comparison of the pressures in the case with ditch and in the case of free space, the absolute values of the pressures are higher in case of the free space.

However, time of the pressure incidence in the given point is longer in the case with ditch, which consequently increases the specific impulse in this case.

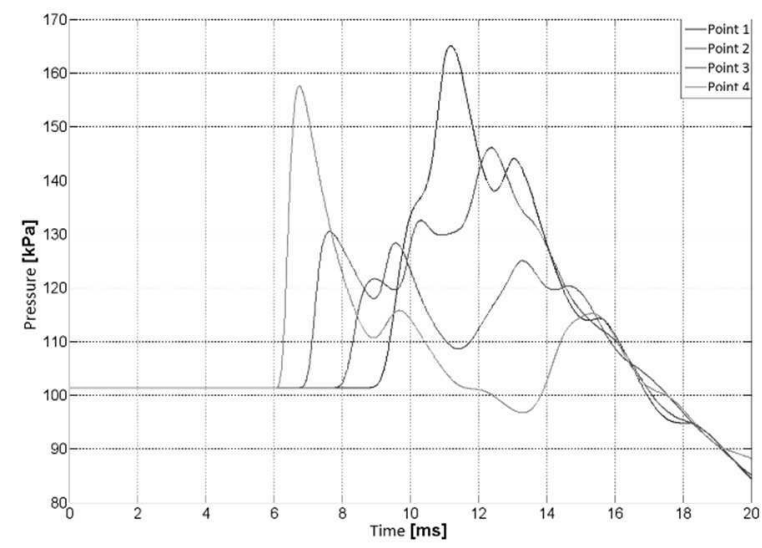

Fig. 12 The course of pressures in the particular point

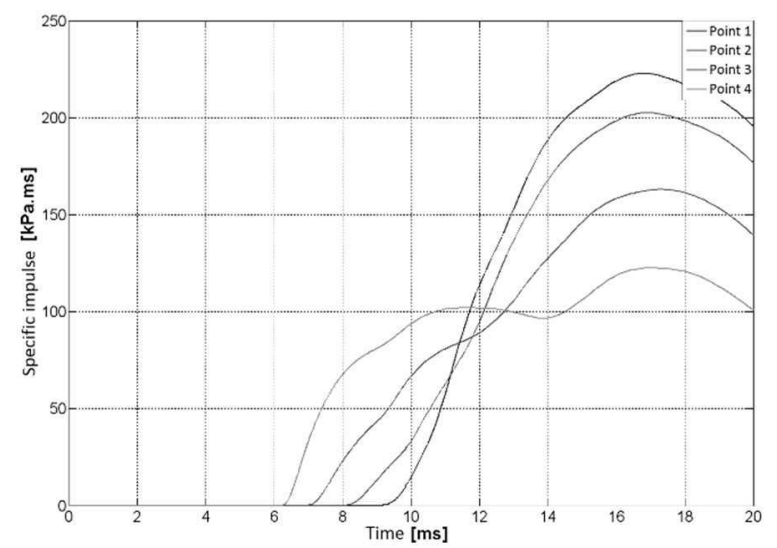

Fig. 13 The course of impulse in the particular points

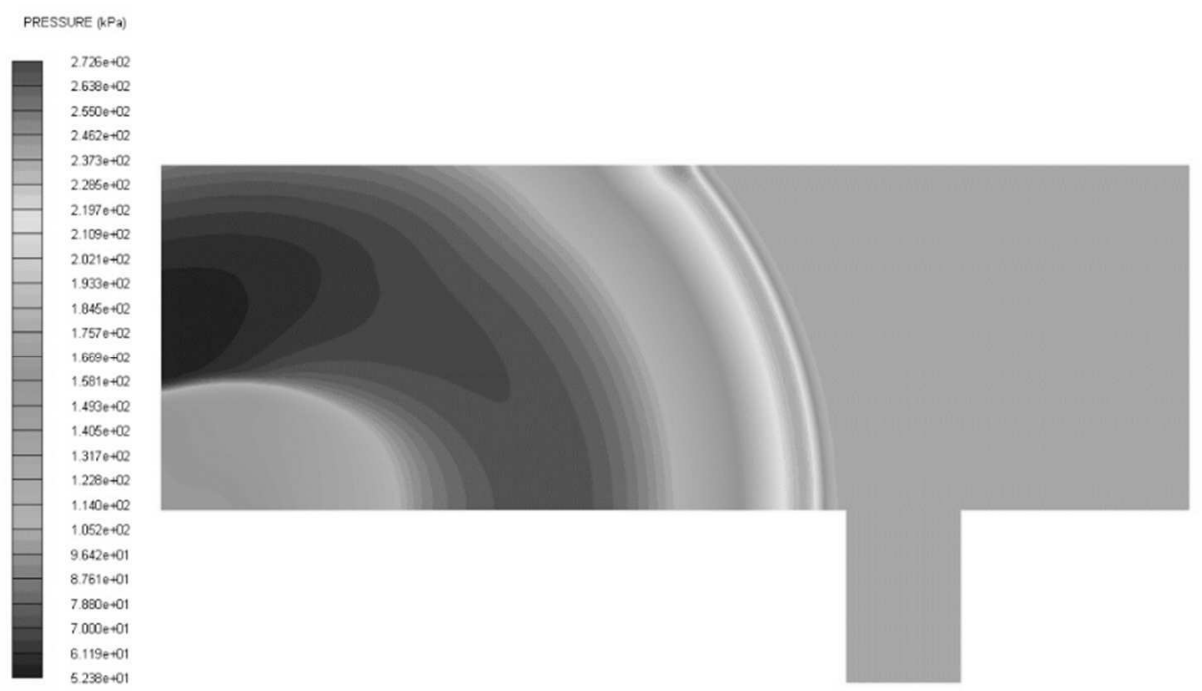

Fig. 14 Propagation of the pressure wave in time $5 \mathrm{~ms}$. 


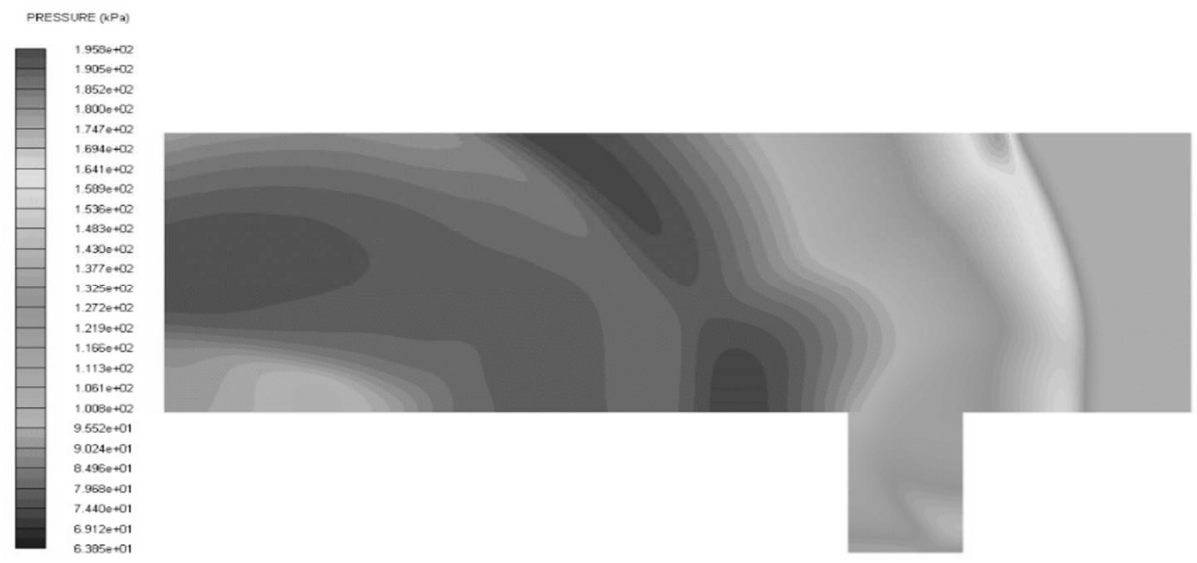

Fig. 15 Propagation of the pressure wave in time $10 \mathrm{~ms}$.

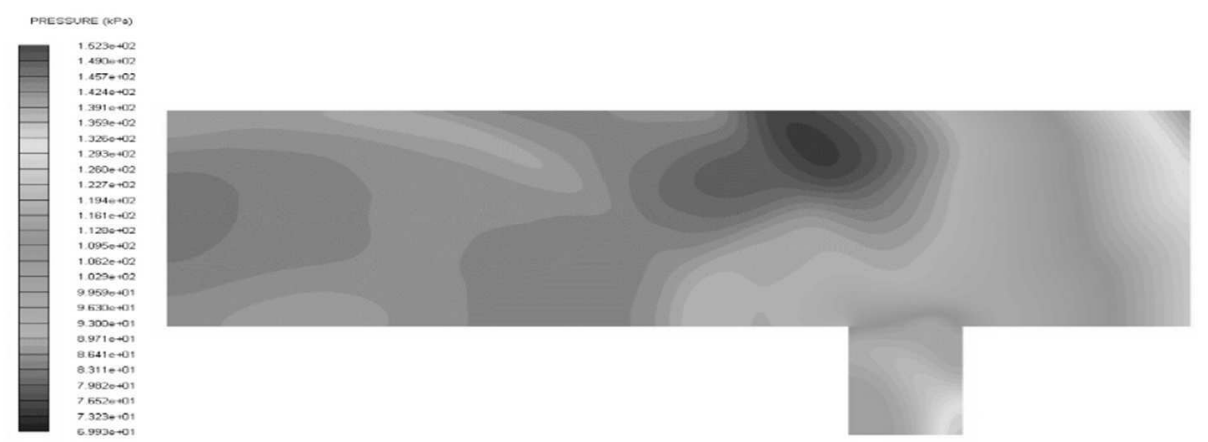

Fig. 16 Propagation of the pressure wave in time $15 \mathrm{~ms}$.

\subsection{Propagation of the pressure wave over pendicular wall}

In the Figure 17, the scheme of the model for calculation of the pressure wave propagation in the space with consideration of the perpendicular wall. The blue area represents Euler mesh. The position of the explosive is marked by red semicircle. The considered explosive had weight $7 \mathrm{~kg}$ of TNT. This model served for obtaining of the essential values of pressure during explosion of described explosive and for propagation pressure wave also in the region with perpendicular wall. Model represents contact explosion with ideal rigid surface, which represents earth ground. The walls of the obstacle are also rigid. The spatial arrangement and boundary condition definitions are similar as previous cases and it is shown in the figure 17.

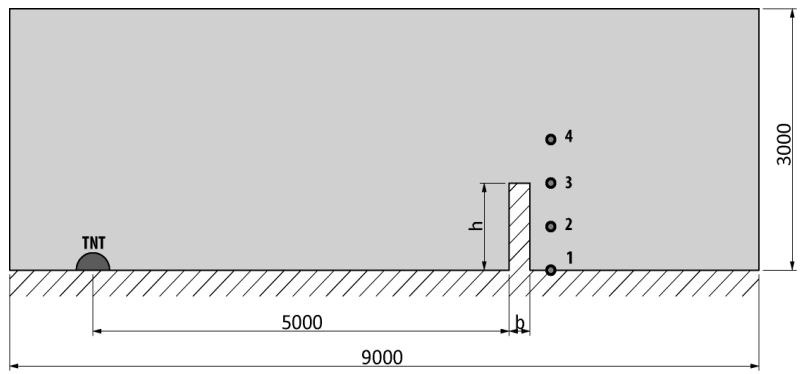

Fig. 17 Scheme of the model with wall

The distance of the perpendicular wall from location of the explosive is $5000 \mathrm{~mm}$. The adjacent side of the wall to the explosion is always at the same distance from location of the explosion. The position of the reverse side of the wall depends on thickness of the wall. At distance of $300 \mathrm{~mm}$ behind the wall from direction of the explosion the check measuring points are considered, in which the time pressure trend was recorded. The displacement of the particular points is $500 \mathrm{~mm}$ in the $\mathrm{z}$ axis direction. The various thicknesses and heights of the wall were considered in calculations, see table 1 By the same method as used in the previous cases, this model is uniformly divided by hexagonal elements with dimensions 50 x 50 x 50 $\mathrm{mm}$. Total number of the elements is from 416000 to 428800 and depends on the volume of the wall.

Tab. 1 Dimension of the wall, see also figure 17.

\begin{tabular}{|c|c|c|}
\hline Wall & $b[\mathrm{~mm}]$ & $h[\mathrm{~mm}]$ \\
\hline 1 & 200 & 1000 \\
\hline 2 & 200 & 1500 \\
\hline 3 & 400 & 1500 \\
\hline 4 & 500 & 1000 \\
\hline 5 & 600 & 1500 \\
\hline 6 & 1000 & 1000 \\
\hline
\end{tabular}

In the figures 18 and 19 , the pressure trends for particular check measuring points and for particular dimensions of wall have been shown. Point 1 is located at surface of the ground and has been shielded by wall for all cases. For that reason, the pressures are approximately same. Differences exhibited in these trends are caused by particular thicknesses of the wall and measuring points are therefore from different displacement from location of the explosion. Less differences can be observed for point 2. From pressure trends is obvious, when considering the specific point, that value of the pressures is 
uniquely affected by height of the wall. The highest difference can be found for points 3 and 4, where the height of the wall creates shield for the measuring place or not.

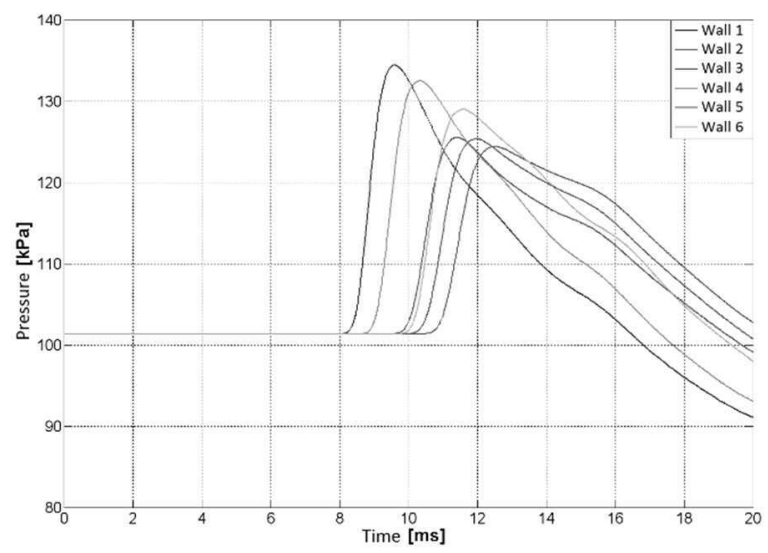

Fig. 18 The course of pressure in point 1 for case with wall

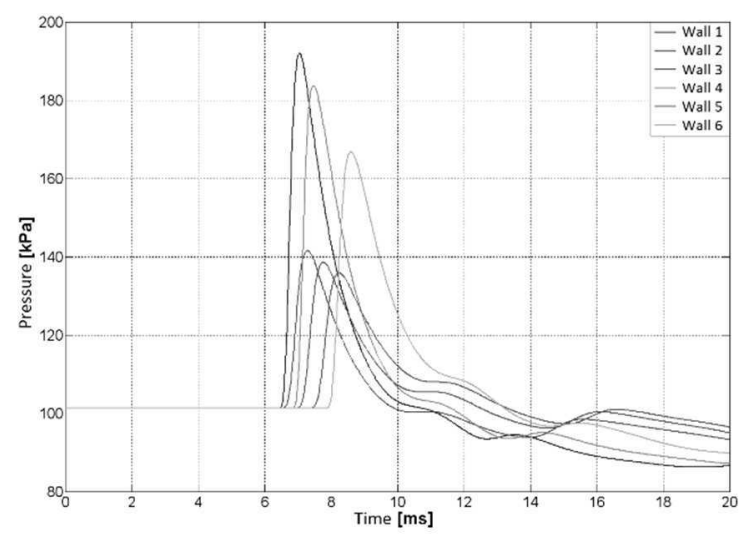

Fig. 19 The course of pressure in point 4 for case with wall

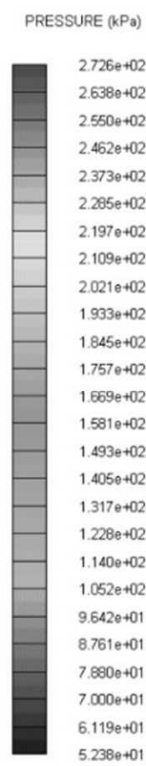

Fig. 21 Propagation of the pressure wave, case with wall, situation in time, $5 \mathrm{~ms}$.

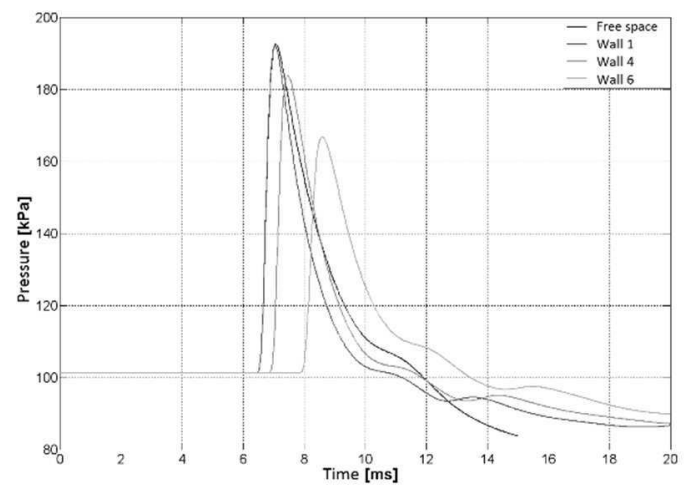

Fig. 20 Comparison of the course of pressure in point 4 for case with wall and case with free space

When the pressure trends in the point 4 are compared for case without obstacle and case with wall of height $1000 \mathrm{~mm}$ (point without shield) and for various thickness (Fig. 20), it can be seen that the maximal values of the pressures are equal for thickness $200 \mathrm{~mm}$. The difference is only in the incidence time of the pressure, which is exhibited in the magnitude of the specific impulse for specific place of impact. From comparison of the specific impulses in point 4 for case of free space and for case with wall 1 , the significant difference of loading appears.

In the figure 21, 22 and 23, the propagation of the pressure wave contours over the wall 1 is shown. In the figure can be found the generation of the reflection wave, which is reflected from adjacent side of the wall and is distributed back to the location of the explosion initialization. Behind the wall, the stepping front of the wave is bent and consequently spread in shape of semi-sphere with center at reverse top edge side. 

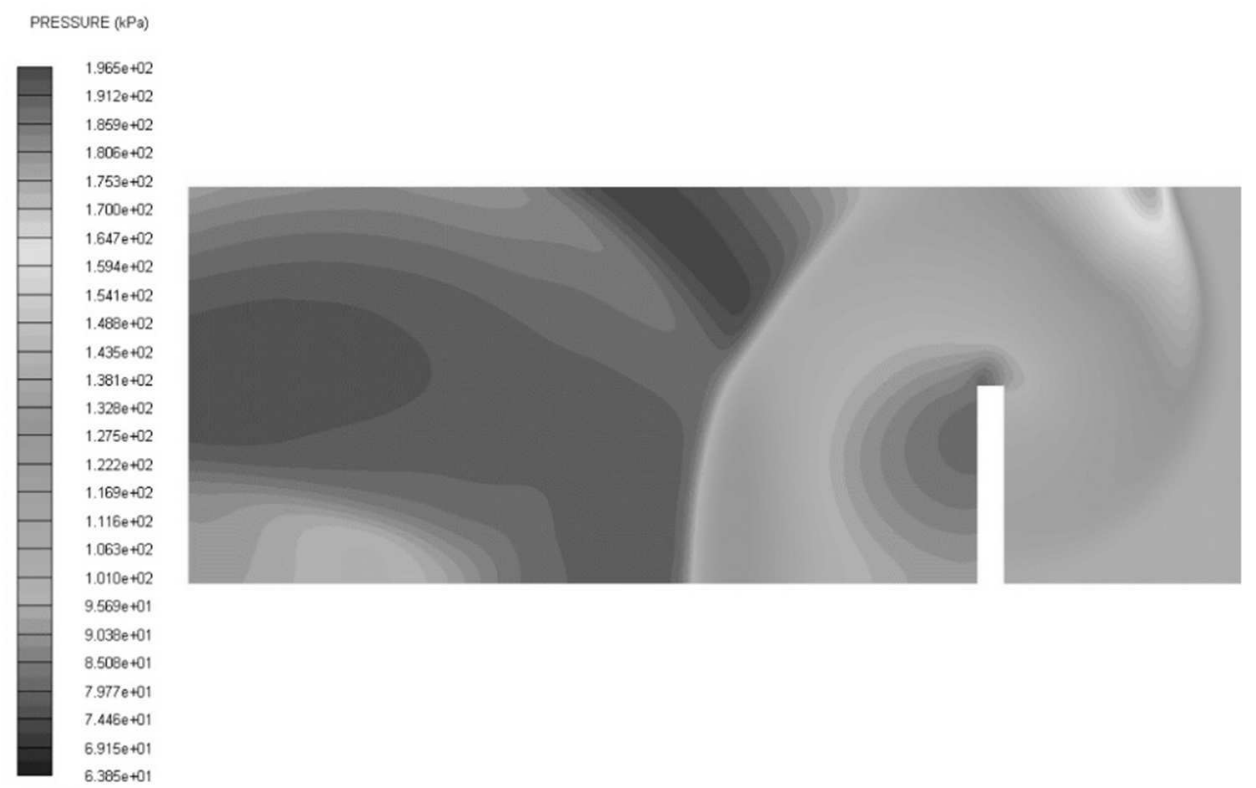

Fig. 22 Propagation of the pressure wave, case with wall, situation in time, $10 \mathrm{~ms}$.

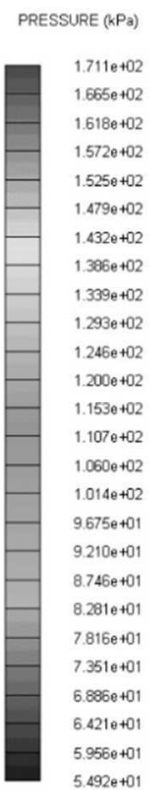

Fig. 23 Propagation of the pressure wave, case with wall, situation in time, $12 \mathrm{~ms}$.

In the previous text, the ideally rigid wall was considered. In the region, where is the location of the supposed obstacle, the boundary of the Euler region is created and at these boundaries the proper conditions are defined. These boundary conditions ensures the ideal reflection of the impulse wave at boundary. This method can be applied for simplification of the calculation only when the propagation of the pressure wave is the main interest and the response to the loading from the explosion of the obstacle is neglected. For this simplification it is not necessary to include into the model the real material properties of the obstacle.

Actually, there is no ideally rigid material. However for the most rigid possible material, which can be applied for real case for creation of the perpendicular obstacle, the transition of the pressure wave at interface wall-air happens. For comparison the model with concrete block was created. Dimension of the block is same with wall 1. From comparison of the pressure trends for investigated point arises, that the difference between trends is practically negligible, which means, that replacement of the concrete block by ideally rigid wall has no effect on the propagation of the impulse wave interaction with perpendicular wall.

\section{Conclusion}

Actual software allows simulation of the behavior of technical object loaded by pressure wave and explosion. It is possible to simulate specified conditions and theirs effect on the object without expensive and time consuming tests. By comparison of the results has been found that, the agreement between theoretical expectations and results of the numerical simulations of the impulse wave 
propagation are practically identical. Based on the performed simulations, it can be stated that used simulations are strong tool for design of the impact loaded structures.

\section{Acknowledgement}

This work was supported by internal grant of Jan Evangelista Purkyňe, Faculty of Mechanical Engineering (UJEP-2018-48-002-2 and UJEP-IGS-2018-48-002-1).

\section{References}

[1] STAREK, L., MUSIL, M., INMAN, D. J. (1997). Updating of non-conservative system using inverse methods. In DETC '97: Proceedings of the ASME International Design Engineering Technical Conferences, Sacramento, pp. 14-17. California.

[2] STAREK, L., INMAN, D.J., MUSIL, M. (1997). Updating of Non-conservative Structure Via Inverse Methods with Parameter Subset Selection. In: Fifth International Congress on Sound and Vibration, Adelaide, pp. 8., South Australia

[3] ZUKAS, J.A. (1990). High-velocity Impact Dynamics. New York, John Wiley.

[4] BUCHAR, J., VOLDŘICH, J. (2003). Terminal Ballistics. Academia Praha, 2003.

[5] SAPIETOVA, A., GAJDOS, L., DEKYS, V. (2016). Analysis of the Influence of Input Function Contact Parameters of the impact force Process in the MSC.ADAMS. Advanced Mechatronics Solution Book Series: Advances in Intelligent Systems and Computing, Vol. 393, pp. 243-253.

[6] DIZO, J. (2015), Evaluation of Ride Comfort for Passengers by Means of Computer Simulation. Manufacturing Technology. Vol. 15, No.1, pp. 1420.
[7] WOLFE, Ch. (2015). The future of simulation. ANSYS Advantage. Special issue: Oil and Gas, ANSYS, Inc., pp. 5-9.

[8] ŽMINDÁK, M., PELAGIĆ, Z., BVOC, M., (2014). Analysis of high velocity impact on composite structures. Applied Mechanics and Materials, Vol. 617, pp. 104-109.

[9] McDOWELLA, D.L. CHOIB, H.J., PANCHALC, J., AUSIND, R., ALLENE, J., MOSTREFF, F. (2007), Plasticity-Related MicrostructureProperty Relations for Materials Design, Key Engineering Materials Vols. 340-341, pp. 21-30.

[10] ŽMINDÁK, M., PASTOREK, P., MOČILAN, M. (2015). Development of the simulation models for pressure wave propagation. In: Proceedings of Dynamics of Rigid and Deformable Bodies (DTDT 2015). Ústí nad Labem.

[11] BRAJA, M. DAS (2008). Advanced Soil Mechanics. Third edition. Taylor \& Francis.

[12] VAVRO JR., J., VAVRO, J., KOVÁČIKOVÁ, P., BEZDEDOVÁ, R., HÍREŠ, J. (2017), Kinematic and Dynamic Analysis and Distribution of Stress in Items of Planar Mechanisms by Means of the MSC ADAMS Software. Manufacturing Technology. Vol. 17, No.2, pp. 267-270.

[13] BAKOŠOVÁ, D. (2018), Dynamic Mechanical Analysis of Rubber Mixtures filled by Carbon Nanotubes. Manufacturing Technology. Vol. 18, No.3, pp. 345-351.

[14] WU, Y., ZHANG, H., LUO, L., XU, Y. (2017) Dynamic Planning for Product Platform and Module Based on Graph Theory. Manufacturing Technology. Vol. 17, No.5, pp. 875-880 